Onkologe 2009 · 15:955-956

DOI 10.1007/s00761-009-1697-x

Online publiziert: 7. Oktober 2009

(c) Springer Medizin Verlag 2009

\author{
U. Wedding $\cdot$ K. Höffken \\ Klinik für Innere Medizin II, Universitätsklinikum Jena
}

\title{
Drängender denn je: Ethik und Onkologie
}

mann und Schildmann daher mit einem Fragezeichen. Sie beschreiben Modelle der Arzt-Patienten-Beziehung unter ethischen Aspekten und stellen Ergebnisse wissenschaftlicher Untersuchungen zu Kontextfaktoren und Präferenzen von Krebspatienten im Rahmen von Aufklärungsgesprächen und Therapieentscheidungen dar.

Meran und Niedersüss-Beke sprechen gar von einer "Autonomiefalle“ in der Betreuung onkologischer Patienten. Um rechtlich relevante Aufklärungsmängel zu vermeiden, werden Patienten sehr umfassend und defensiv aufgeklärt, was zu Verängstigung und Überforderung führen kann. So kann die zum Nutzen der Patienten gedachte Autonomie manchmal in der Not der Krankheit auch zu einer Falle werden. Wie in dieser Situation Fehlentwicklungen zu vermeiden sind, führen die Autoren aus.

Während die beiden erstgenannten Arbeiten die Auswirkungen auf den Patienten im Fokus haben, analysiert Maio die Auswirkungen auf die Arzt-Patienten-Beziehung. Er sieht die Gefahr in der Orientierung der modernen Medizin allein an der Ökonomie und in der Befolgung von Klientenwünschen. Im Gegenzug plädiert er dafür, Autonomie und Fürsorge miteinander in Verbindung zu bringen, die soziale und karitative Dimension der ArztPatienten-Beziehung aufrechtzuerhalten und die Onkologie nicht der Marktfähigkeit unterzuordnen.

Ad 2: Ethische Entscheidungen in der Onkologie können durch strukturierte ethische Fallbesprechungen unterstützt werden, an denen alle Berufsgruppen und medizinischen Fachdisziplinen teilnehmen, die an der Versorgung des Patienten beteiligt sind. Inhaltlich sollte sich das Gespräch an den vier klassischen medizinethischen Prinzipien Wohltun, Nichtschaden, Respekt der Autonomie und Gerechtigkeit orientieren. Wie dies zum Wohle aller Beteiligten - Patienten, Angehörige und Betreuende - erfolgen kann, stellen Marckmann und Mayer in ihrem Beitrag dar.

Ad 3: In der klinisch-onkologischen Forschung können Patientenfürsorge und Erkenntnisgewinn im Konflikt stehen. Worin diese Konflikte bestehen und wie eine Lösung aus ethischer Sicht möglich ist, stellen Illhardt und Unger an den Punkten Vulnerabilität, Patienteninformation, Interessensbalance („equipoise“), Monitoring der Datensicherheit, Publikation und der Erforschung palliativmedizinischer Konzeptionen dar.

Ad 4: In den zurückliegenden Jahren wurden viele neue Medikamente zur Therapie onkologischer Erkrankungen zugelassen. Die Entwicklungskosten, bis diese Medikamente zur Zulassung kamen, waren oft sehr hoch. Die Preise nach der Zulassung sind es ebenso. Ein Teil der Substanzen wurde für eine Nischenindikation zugelassen, konnte im Verlauf aber ein breiteres Wirkspektrum nachweisen. Ein Teil kann aufgrund des exzellenten Wirkungs- und Nebenwirkungsprofils als Therapie über einen viel längeren Zeitraum gegeben werden als im Rahmen der Preiskalkulation angenommen. Gleichzeitig wirken viele dieser neuen, innovativen, aber auch teuren Substanzen nur bei einem Teil der behandelten Patienten.

Die Frage, für welche Patienten diese Medikamente aus der Gemeinschaft der Versicherten finanziert werden sollen, ist auch eine ethische Frage. Knöpfler fragt heit“" zu ihrem Beitrag versehen Schild- 
dies aus Sicht des Medizinethikers mit dem Blick über den deutschen Tellerrand hinaus. Ludwig et al. stellen die Situation in Deutschland dar. Erforderlich sind Studien nach erfolgter Zulassung, die eine Nutzenbewertung ermöglichen. Sie ist Grundlage einer notwendig werdenden Priorisierung trotz anderslautender Äußerungen gewählter Volksvertreter.

Ad 5: Unter der Überschrift „Genetische Krebsdiagnostik - Fluch oder Segen für die Patienten?" zeigt Propping, dass das Wissen um ein hohes Krebsrisiko eine hohe Belastung darstellt. Die persönliche genetische und klinische Beratung ist daher Voraussetzung für eine gute Compliance eines Patienten, der ein hohes Krebsrisiko hat. Wie verschiedene Studien zeigen, wird allerdings selbst ein positiver prädiktiver Test als weniger belastend empfunden als eine fortbestehende Unsicherheit über die eigene Risikosituation.

Die Schwerpunktherausgeber stellen mit diesem Heft den onkologisch Tätigen eine fundierte Darstellung einiger aktueller Themen an der Schnittstelle von Ethik und Onkologie vor. Im Licht der gesellschaftspolitischen wie auch ärztlichen Diskussion sind sie wichtige Beiträge mit Antworten auf drängende Fragen.

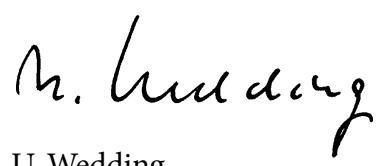

U. Wedding

Für die Herausgeber des Schwerpunkthefts

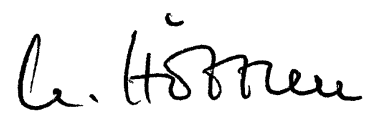

K. Höffken

Für die Herausgeber

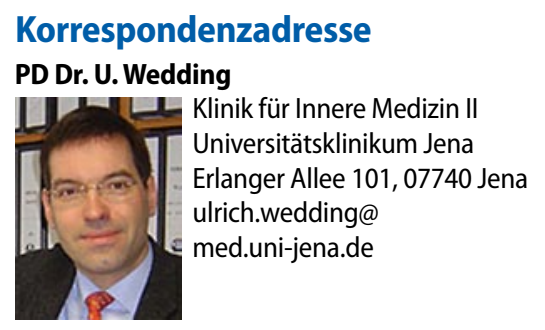

Interessenkonflikt. Der korrespondierende Autor gibt an, dass kein Interessenkonflikt besteht.

\section{Arthur Walpole Preis 2010}

Der Arthur Walpole Preis wird von der Deutschen Krebsgesellschaft alle 2 Jahre an die beste klinische Studie verliehen. Studienleiter, die eine richtungsweisende klinische Studie erfolgreich abgeschlossen und publiziert haben, können sich bewerben.

Für die Auszeichnung kommen grundsätzlich klinische Studien aller Phasen in Betracht. Der klinischen Studie muss ein qualitativ hochwertiges Studienprotokoll zugrunde liegen. Kriterien für die Qualitätsbeurteilung sind das innovative Konzept mit richtungsweisender Fragestellung und gutem Studiendesign (z.B. Gütesiegel A der Deutschen Krebsgesellschaft). Besonderer Wert wird auf die Qualität der Studiendurchführung und die Berücksichtigung von Good Clinical Practice Kriterien gelegt. Die Ergebnisse der Studie müssen in einer Publikation beschrieben sein, die internationalen Kriterien entspricht und in einer hochrangigen Zeitschrift mit, peer review' innerhalb der vergangenen zwei Jahre veröffentlicht worden sein.

Der Arthur Walpole Preis ist dem Entdecker des Tamoxifen gewidmet und wird gestiftet. Das Preisgeld von insgesamt 10.225 EURO wird aufgeteilt zwischen dem Studienleiter der ausgezeichneten Studie (5113 $€)$ und den Vertretern der beiden am Gelingen des Projektes maßgeblich beteiligten Zentren (je $2556 €$ ).

Über die Vergabe entscheidet das Preiskomitee, bestehend aus den 14 Mitgliedern der Kommission Klinische Studien in der Onkologie der Deutschen Krebsgesellschaft und zwei Vertretern des Stifters. Alle Studienleiter von Studien, die die Ausschreibungskriterien erfüllen, können sich direkt bewerben oder auch von einem Mitglied der Deutschen Krebsgesellschaft vorgeschlagen werden. Der formlosen Bewerbung ist ein Exemplar des Studienprotokolls und ein Sonderdruck der Abschlusspublikation beizufügen. Die Preisverleihung wird auf dem Deutschen Krebskongress vom 24. - 27. Februar 2010 in Berlin erfolgen.
Bewerbungen sind bis zum 31. Oktober 2009 (Datum des Poststempels) zu richten an: Deutsche Krebsgesellschaft e.V. Herrn Professor Dr. Ulrich Keilholz Vorsitzender der Kommission Klinische Studien in der Onkologie Straße des 17. Juni 106-108 10623 Berlin 